\title{
Index Volume 4
}

(Numbers followed by asterisk refer to Society Abstracts)

Aagenaes, O. 377*

ABILDGaARD, C.F. 464*

Absorption, jejunal 477*

AcETo, T., Jr. 459*

Acid-base homeostasis $447 *$

Acid-base metabolism 205*

Acid mucopolysaccharide 440*

Acidosis 470*

Acidosis, metabolic 451*

ACTH 207* 459*

ADAM, P.A.J. 120

Adams, M. S. 474*

Adenine nucleotide 465*

Adenoids 371*

$S$-Adenosyl-L-methionine 454*

Adipose tissue 135, 268, 453*

Adolescence 268

Adolescents 175

Adrenal 457*

Adrenal, $\alpha$-inhibition 456*

Adrenal dysplasia 257

Adrenal, function 458*, 459*

Adrenal hyperplasia, congenital $458^{*}$

Adrenal hyperplasia, lipoid 455*

Adrenocorticosteroid 462*

AdRIANZEN T., B. 375*

Agathopoulos, A. 203*

Age 63

AICARDI, G. 206*

Airway obstruction 478*

Aksu, A. 480*

Alagille, D. 215*

Alanine 451*

Alcoholism, maternal 474*

Aldolase, liver 206*

Aldosterone 145, 455* 457*

Alkaline phosphatase $450 *$

Allergy 435*

Alpha ${ }^{1}$-antitrypsin $477 *$

AL-Ubaid, Y.Y. 448*

Ambrus, C. M. 82

Ambrus, J.L. 82

Amino acid metabolism 209*, $238,447 *, 453^{*}$

Amino acids 216*

Amino acids, metabolism 216*

Aminoaciduria 480*

Aminoglutethimide 455*

$\gamma$-Aminolevulinic acid $216^{*}$

Ammann, A. J. 435*

Ammonia 446*

Ammonia excretion 398

Amniocentesis $377 *, 440 *$

Amniotic fluid $372 *$

ANCEs, I. G. 455*

Anderson, C. M. 212*

Androgens 468*

Anemia 280, 318

Anemia, aplastic 468*

Anemia, congenital hypoplastic 217*

Anemia, hemolytic 209*, 217*

Anemia, hypoplastic 209*
Anencephaly 257

Anesthesia 471*

Aneuploidy, XYY 205*

ANTHONY, B.F. 460*

Anthony, C. L., Jr. 473*

Antibiotics $472 *$

Antibodies 71, 435*, 436*

Antibody $461^{*}, 500$

Antibody, rubella 372*

Antielastase 477*

Antistaphylococcus $463^{*}$

Apostolico, R.J. 472*

ARBIT, J. 378*

Ararlia, R. A. 445*

Argininosuccinate synthetase 203*

ARIAS, I. M. 476*

ARMsTrong, D. 461*

Aspartate transcarbamylase 55

Aspin, N. 478*

Aspiration pneumonia $479 *$

Assemany, S. R. 441*

Asthma $435^{*}, 436^{*}$

Ataxia telangiectasia $435^{*}, 441^{*}$

ATPase 445

Aurd, P. A. M. 479*

Autoimmunity 435*

Avery, M.E. 201*

AvRuskin, T. 456*

Ayoub, E.M. 460*

BÄGHr, T. 213*

Bacteremia 464*

Bacterial endocarditis $463 *$

Bacterial infection $461 *$

Bacteriuria 500

BAEHNER, R. L. 476*

BAER, H. 472*

BAERLOCHER, K. $447 *$

BAIN, H.W. 477*

BAKER, L. 295, 377*

BAKKEN, A.F. 214*

BANK, W. 480*

Baptistal, R. 214*

BARNESS, L.A. 451*, 453*, 480*

BARRETT, C.T. $443^{*}$

BARRETT, F.F. 463*

Bartsocas, C.S. $451^{*}$

BartTer, F.C. $457 *$

Battaglia, F.C. $445 *$, 471 *

BAU, S.K. 478*

Baublis, J.V. 482*

BAUM, D. $456 * 473 *$

BAYARD, F.J. 455*

BeaudRY, P.H. 378*

Becattini, F. 464*

BECK. J. 378*

Behavior 475*

Behavior, family 475*

BEITINS, I.Z. 129

Bellañx, J.A. 405, 435*, 462*

BENSON, L. 194

Berkovich, S. 462*

BERLIN, C.M. $377 *$
BERNFIELD, M. 440*

Bertrand, J. 145

Blc 71

Bettens, W. 206*

BIGKeL, H. 217*

BIER, H. 212*

Bile 377*

Bile acid metabolism 214*

Bile acids 262

Bilgic, A. 444*

Biliary atresia 262

Biliary obstruction $476 *$

Bilirubin 214*, 465*, 481*

Binoux, M. 207*

Binz, H. 213*

Birth weight 14

Blanc, W. 257, 375*, 473*

Blaufox, M.D. 450*

Bleeding time 229

BLONSKY, E. R. 378*

Blood 379*

Blood, cord 436*

Blood data 103

Blood glucose 202*

Blood pressure 63

Blood substitute $464^{*}$

Blumenfeld, O. 476*

BoAT, T.F. 457*

BODÁ, D. 202*

BoDE, H.H. 458*

Body temperature 63

BogGs, T.R. $481 *$

Bone age 25, 36

Bone marrow 468*

Bonglovanni, A. M. 175, 455*

Bonnassiev, M. 204*

Bonnassieux, M. 214*

BOWMAN, R.L. 470*

Boys 205*

BRADLEX, K.H. 379*

BRADY, J.P. 472*, 478*

Brain 96, 379*, 471*, 480*, $481 *, 482 *$

Brandt, N. J. 209*

BRIESE, F.W. $460^{*}$

BRODSKY, S. J. 445*

Bromide space 14

Bronchitis 208*

Bronchopulmonary dysplasia 202*

BROSS, I. D.J. 82

Brown, A. K. 466*

Brown fat 453*, 472*

BRUNell, P.A. $462 *$

Brunette, M.-G. 451*

Brush border, intestinal 477*

Bryan, G.T. 63

BUCKLEY, R.H. 436*

Burgro, G.R. 207*

Burkitt's tumor 204*

BurR, I.M. 25, 36

Bush, H. L. 444*

Butron, L. N. 465*

BUYLE, M.L. 214* 
Calcagno, P. L. $446 *$

Calcium 202*, 210*

Caldwell, B. M. 374*

Calldahan, J. 438*

Calorie restriction 157

Campbell, A.G. M. 443*

CAMPBEll, P. 480*

Canent, R. V. 444*

CanTz, B.E. 405, 462*

Carbohydrate absorption 477*

Carbohydrate metabolism 373*, $377 *$

Cardiac defects $445^{*}$

Cardiac output 7, 443*

carditis, rheumatic 207*

Care, pediatric $371^{*}$

Carotene $475^{*}$

Cartilage 202*

CASSADY, G. 14

Cassell, S. 378*

Casteels-van Daele, M. 206*

Catch-up growth $459^{*}$

Catecholamines 203*, 216*, 295

Catechol- $O$-methyltransferase $203^{*}, 454^{*}$

Catheters, polyethylene 464*

CAUL, J. 465*

Celander, O. 201*

Celiac disease 214*

Geliac syndrome 476 *

Cell hybridization $376^{*}$

Cellular response $437^{*}$

Central nervous system 203*, $378 *, 379 *, 481 *, 482 *$

Central nervous system damage $481 *$

Gнавот, A. 373*

Chacko, C. M. 376*, 439*

Ghan, J.G. $447^{*}$

Ghapman, E. M. 374*

Chapman, S. S. 448*

Ghase, H.P. 96

Cheek, D.B. 135, 157, 268

GHen, S. Y. 457*

CHeRRY, R. B. 479*

Child care $373^{*}, 374 *$

Children 165, 175, 208*, 216*, $262,378^{*}, 474 *, 478 *$

Children, abused neglected $474 *$

Chloramphenicol 442*

Gно, С. T. 463*

Cholestasis 215*, 377*

Cholesterol 215*

Gifristian, J. C. 439*

Ghromosomes 204*, 205*, 377*, $439 *$

Chronic granulomatous disease $405,462 *$

GHuck, G. 452*

Circadian rhythms 63

Circulation 443*

Circulation, cerebral 471*

Circulation, umbilical 471*

Cliark, L. C., Jr. 464*

Clements, R.S.,Jr. 466*

Cleveland, W.W. 76

Glyde, W.A., Jr. 460*

Coagulation 229, 464*

Cochran, W. 373*
Cohen, M. 462*

CoHen, M. I. 476*

Cohen, M.M. 375*

Cold stress 473*

Collagen 135

Collagen, skin 206*

Collier, A. M. 460*

Collipp, P.J. 457*

Colostral cells 71

Colwill, J.R. 471*

Coma, hepatic 207*

Community health program $373^{*}$

Compliance, lung 202*

Condon, A. $371^{*}$

Congenital adrenal hyperplasia $145,309,380 *$

Congenital anomalies $377^{*}$

Congenital disease $454 *$, 458*, $465 *$

Congenital rubella $372 *$

ConLy, P.W. 76

Cook, C.D. 478*

CoOper, G. 443*

COOPER, L.Z. 372*

Copper deficiency 244

Copper metabolism 217*

Corbeel, L. M. 206*

Corbx, D. G. 379*

Cordano, A. 135

Cornblath, M. 451* 472*

Coronary artery disease $440 *$

Corrigan, J.J. 464*

Corticosteroids 201*

Cortisol 129

Cortisone 459*

Cote, M.L. 449*

CotTe, J. 214*

CourPotin, C. $472 *$

Courtecursse, V. 215*

Goussons, H. 451*

Cox, D.E. 467*

CRANCE, J.P. 202*

CRAWFORD, J.D. 374*, 458*

Creatinine 135

Grigler-Najjar syndrome 214*

Grocker, J.F.S. 448*

Gromroy, H. L. 468*

CsER, A. 202*

Cuderman, B. 377*

Cuisinier-Gleizes, P. 202*

Cushing's disease 456*

Gyclic AMP 456*, 458*

Cystathionase 205*, 337, 442*

Cystathionine $442 *, 453 *$, 454*

Cystathionine synthetase 337

Cysteine 454*

Cystic fibrosis of the pancreas $262 *, 456 *, 457 *, 475 *, 478 *$

Cystine 379*, 454*

Cystine dependency 476*

Cystinosis 379*

Cytochalasin b 44.1*

Cytochrome oxidase $328,442 *$

Cytomegalovirus 461*, 462*

DAENTL, D. L. $442 *$

DaJanI, A.S. 448*
Dallatre, L. 451*

Dallman, P.R. 244

Damato, A. N. 445*

DANCIS, J. 345

Dandy-Walker syndrome 481*

Davidson, M.H. 295

Davidson, W. 318

Davis, C.W. G. $460 *$

Davis, K.L. $472 *$

DAVIS, S.D. 441*

Deafness $372 *$

DEgENHART, H.J. 309

DE Groot, C. J. 238

Deinard, A.S. 467*

DeLemos, R.A. 129, 201*

Delinquents 205*

Delivoria-Papadopoulas, $M$ $444 *, 470 *$

DeLuCA, H.F. 451*

Denning, G. R. 475*

Deoxyadenosyl-B $\mathrm{B}_{12} 374 *$

Dermatoglyphics $441^{*}$

Development 25, 36, 379*

Development, idiopathic isosexual precocity $458^{*}$

Development, retardation $459^{*}, 460 *$

Developmental biochemistry $89,96,203 *, 328,352,442^{*}$, $446^{*}, 447 *, 453 *$

Developmental pharmacology $379 *, 443 *, 471 *, 480 *$

Developmental physiology 7, $165,444 *, 446 *, 447 *$

Diabetes insipidus $457 *, 458 *$

Diabetes, maternal $472 *$

Diabetes mellitus 219*, 456*, 477*

Diagnosis, prenatal $372 *$

Diarrhea 210*, 476*, 477*

Diarrhea, congenital, chloride wasting $214^{*}$

DiChiro, G. 459*

Digitalis $445^{*}$

Dihydro-orotase 55

Dihydrotestosterone $455^{*}$

DiMauro, S. 371*

Dimethyl sulfoxide 493

Dipeptidases, intestinal 211*

Dipeptides 477*

Diphenylamine 448*

Diphenylhydantoin $455^{*}$

2,3-Diphosphoglycerate 444*

2,3-Diphosphoglycerate, erythrocyte 467*

Diphosphorylase kinase 452*

DI SANT'Agnese, P.A. 457*

DNA $135,157,268,435^{*}$

DNA antibodies $435^{*}$

DNase II 493

DODGE, W.F. 450*

DoershuK, C. F. 165

DONHOFFER, H. 202*

Donnadieu, M. 207*

Doppman, J. $470 *$

Dorfman, A. 372*

DownING, S.E. $443 *$

Down's syndrome 187, 439*, 467* 
524

Downs, T.D. 165

Drachman, R.H. 461*

Drash, A.L. $457 *$

Drug resistance $472 *$

Duc, G. V. 467*

Dumpit, F.M. 472*, 478*

Durant, J. L. $454 *$

Dwarf 459*

Dwarfism, hypopituitary 480*

Dwarfism, primordial 207*

Dysmature 202*

EBADr, M.S. 187

EberLeIN, W.R. 175, 455*

Eck, E. 202*

Ecology, children 475*

Ecology, infants 474*

Ecology, sociological 473*

Eczema 435*

Edelmann, C.M.,Jr. 7, 450*

Edema, pulmonary, hemodynamic 479*

EDWARDS, N.K. 472*

EgGermont, E. 206*

Eisner, G. M. 446*

EitZMAN, D. V. 472*

Electromyography 286

ELLER, J. 436*

Embryogenesis 55

Embryo, mouse 375*

EMmaNouilides, G. C. 470 *

Emphysema 477*

Endocardial fibroelastosis $377^{*}$

ENGEL, K. 447*, 467*

Enterokinase 212*

Enterokinase deficiency 211*, $476^{*}$

Enuresis 450*

Enzyme 206*, 377*, 438*

Enzyme activity 328

Enzymic defects 238

Enzyme induction 203*

Enzymes 203*, 442*

Epinephrine 295

EPstein, C.J. $442 *$

Erythroblastosis 217*, 280, 373*

Erythrocyte 217*, 456*, 465*, $466 *, 467 *, 468 *$

Erythrocyte cation transport defect 478*

Erythrocyte, morphology 466*

Erythrocytes 372*, 405

Erythrocytes, sodium flux $478 *$

Erythropoiesis 468*

Erythropoietin 468*

ESCHENBACH, G. 493

Escherichia coli 213*, 500

Ethacrynic acid 478*

Eveland, W.C. 463*

Exchange transfer 207*

Exchange transfusion 481*

Exfoliation 378*

Extracorporeal support 207*

Factor II 203*

Factor VII-X 203*

Factor XIII 82

FagnAN, L.J. 440*

FALLETTA, J.M. 437*
Index Volume 4

Familial protein intolerance 209*

FANARoff, A. $473 *$

Fat transport, intestinal $213^{*}$

Fatty acid biosynthesis $480^{*}$

Fatty acid metabolism 466 *

Fatty acids 352

FAxelius, G. 469*

Feeding, gavage 472 *

FERnandes, J. 206*

Fernbach, D.J. 437*

FERrier, P.F. 205*

FERRIER, S.A. 205*

FERRIERI, P. 448*

Fertilization, in vitro $375^{*}$

Fetal 211*

Fetal homeostasis 345

Fetal transfusions 373 *

Fetal virilization $458^{*}$

Fetus 129, 201*, 203*, 371*, $436 *, 440 *, 441 *, 442 *, 443 *$, $445 *, 457 *^{\prime}, 460 *, 470 *$, 471*'

Fibrin 229

Fibrinolysis $372 *$

Fibrin split products $372 *$

Fibrin-stimulating factor 82

Fibroblasts 455*

FIELD, M. 477*

Fine, E. $472 *$

Fine, R.N. 449*

FireMAN, P. 446*

Fisher, D. A. 457*

Fleming G.A. $451^{*}$

Florman, A. L. 372*

Fluorocarbon $464 *$

Folate metabolism 412, 422

Folic acid $450^{*}, 453^{*}$

Follicle stimulating hormone $25,36,456 *$

FONDU, P. 209*

FRANĢOIs, R. 206*, 214*

Frasier, S.D. $459 *$

FREDRICKSON, D.S. 439*

Free fatty acid $202 *, 219^{*}$, $472 *, 473 *$

FRESCO, R. 454*

FRÉZAL, J. 219*

Friedman, S.B. 462*, 474*

FRIEDMAN, W.F. $443^{*}$

FRUCTOSE 206*

Fructose-1,6-diphosphatase 377 *

Fujmoto, W.Y. 452*

Furosemide $448 *$

Galactose 211*

Galactose 1-phosphate uridyl

transferase $376 *, 439 *, 452 *$

Galactosemia 439*, 452*

$\beta$-Galactosidase 438*

GALL, G. 445*, 456*

GALOFRE, A. 55

Gangliosidosis 438*, 451*

Ganz, R. 202*

GARDNER, J. 194

GARDNER, J.D. 478*

GARDNER, L.I. $441 *$

GarReTtSON, L. K. 455*

Gastroenteritis, Escherichia coli $213^{*}$
GATTI, R. 206*

GatTi, R.A. 467*

GAULL, G. 205*, 337, 442*, $453^{*}$

Gelband, H. 444*

GENel, M. 447*

Genetic disease $374 *, 376 *$, $437 *, 438 *, 439 *, 440 *$ $441 *, 450 *, 476 *, 481$ *'

GenTz, J. $216^{*}$

Gerald, P.S. 438*

Ghadimi, H. 204*

GIBSON, L. E. 478*

Gibson, M.S. 379*

GiNGELL, R.L. 451*

GinsBerG-Fellner, F. 373*

GrRARD, F. 207*

Grtzelmann, R. 213*

GJEssing, L. $216^{*}$

GLADTKE, E. 218*

Glasgow, L.A. 378*, 462*

GLENN, B. L. 465*

Glomerular filtration rate 7

Glomerular nephritis $448^{*}$

Glomerulonephritis $376^{*}$

Glomerulus, basement membrane 448 *

GLORIEUX, F.H. 450*

Glucagon 120, 206*

GLUCK, L. 352

Gluconeogenesis $377 *$

Gluconeogenesis, renal 446*

Glucose 46, 120, $211^{*}, 446^{*}$, $466^{*}$

Glucose, blood 295, 472*

Glucose metabolism 202*, $379 *, 471^{*}$

Glucose 6-phosphate 206*

Glucose 6-phosphate dehydrogenase $405,462 *$

Glucosuria renal 211*

Glucuronyltransferase 214*

$\gamma$-Glutamyl transpeptidase 476 *

Gluten 214*

Gluten enteropathy 476*

Glycerol 453*

Glycine 238

Glycogen 204*

Glycogenosis $206 *, 452 *$

Glycolysis $371^{*}, 465^{*}$

Glycolysis, renal 448*

GOLD, E. 460*

GoldMAN, A.S. 71, 455*

Gonadal dysgenesis 36

Gonads 175, 309

Good, R.A. 463*, 467*

GoOdman, J.R. 244, 378*

GoRDIs, L. 371*

GORDON, D. 473*

Gotof, S.P. 437*

GotTo, A. M. 439*

GOURLEY, B. 476*

Graham, C. B. 440 *

Graham, G. G. 135, 375*

GraHAM, T.P. $444^{*}$

Gram-negative bacteremia 461 *

Gram-negative bacteria 472 *

Granulopoiesis $467 *$

GRAVE, G.D. 481* 
Graven, S. N. 470*

GRAW, R. G., Jr. 461*

Green, O. C. $458^{*}$

GREENBERG, R. E. 446*, 453*

GREENE, M.L. 452*

Gregory, G. A. 469*

GRESHAM, E. L. 445*

GRIPPO, J. 480*

Grossman, B.J. 459*

Grota, L.J. 462*

Growth 318, 375*, 449*, 474*

Growth, accelerated 440*

Growth failure $376^{*}$, 449*, 476*

Growth hormone 457*

Growth hormone, human 459*

Growth retardation 14, 459*

Growth, skeletal 440*

GRUBER, H. 473*

GRUMBACH, M. M. 25, 36, $380^{*}, 480^{*}$

Grushkin, C. $449 *$

Gruskin, A. B. 7, 449*

GUTBERLET, R.L. 469*

GYeRMEK, L. 471*, 480*

HADDAD, Z.H. 436*

HADDOW, J.E. 286

HADORN, B. 211*

HAGEMEIJER, A. M. 438*

HaGHBin, M. 461 *

HALLER, J.S. 481*

HALLORAN, K.H. 443*

Halstead, S. B. 373*

HALVORSEN, S. $216^{*}$

Hamilton, W.K. 469*

Handelsman, D.G. 437*

HaNel, H. 209*

HANLEY, W.B. 318

HAOUR, F. 145

HARLEX, B. M. 458*

HarRis, R. C. 337

Hasselmeyer, E. G. 472*

Hathaway, W.E. 229

HaWorth, J. G. 476*

HAYEK, A. $374 *$

Hayles, A. B. $459 *$

Hazel, B. 451*

Heagan, B. 467*

Health care $474^{*}$

Heart 244, 443*, 444*

Heart, congenital disease 444 , $445 *, 467 *$

Heart failure $444 *$

Heart, function $445 *$

Heart, hypertrophy $444 *$

Heart muscle metabolism 244

Heart-reactive serum factors 207 *

Heart, rhythm 444*

Heart, sounds $201 *$

Heat loss, radiant $473^{*}$

HeAth, C. W., Jr. 437*

HEIM, L. $463^{*}$

HEIM, T. 202*

HELBOCK, H.J. 455*

Hemodialysis, chronic 449*

Hemoglobin 217*, 467*

Hemoglobin, fetal 466*, 470*

Hemoglobin SS disease $372 *$
H. influenza, type b $376^{*}$

Hempler, S. 463*

Heparin 464*

Hepatic glycogen storage disease $206 *$

Hepatitis 207*, 262, 468*

HeRcz, A. 477*

Hereditary spherocytosis $465 *$

Herpes zoster 462*

HERSGHKOWITZ, N. 203*

HEYMANN, M.A. 443*

Hilgartner, M.W. 468*

HiLl, D.E. 135

Hiltunen, P. 208*

His bundle $445^{*}$

Histamine $437 *$

Histiocytosis X 457*

Hobel, G.J. 457* 470*

HofFMAN, B. F. 444*

Hollerman, G. E. 446*

Holliday, M.A. 449*

Holsclaw, D.S. 456*

Hol, A.B. 157

Holtzapple, P.G. 447*

Holzel, A. 213*

Hommes, F. A. 238

Homocystinuria 205*, 453*, 454*

Homolanthionine 205*, 453*

Homoserine 454*

Hon, E. H. 472*

Hong, R. 435*

Hormone 458*

Hormones 129, 309

HoRnADy, G. 412, 422

Horstmann, D. M. 373*

Hotchin, J. 194

Hoy, , W.F. 480*

HsIA, Y.E. 439*

Hug, G. 452*

Hursman, T.H.J. 466*

Human colostrum 71

Human respiratory epithelium $460 *$

Hunter's syndrome $372 *$

Hurler's disease 438*, 451*

Hurler's syndrome $372 *$

HÜRTER, P. 217*

Hyaline membrane disease $467 *, 469 *, 479 *$

Hybridization, DNA 438*, $439 *$

Hydrocephalus $481 *$

Hydrogen peroxide hemolysis $476 *$

25-Hydroxycholecalciferol 451*

21-Hydroxylase $145,458 *$

21-Hydroxylase deficiency $380 *$

p-Hydroxyphenylpyruvic oxidase 337

$3 \beta$-Hydroxysteroid dehydrogenase $458 *$

Hyperalaninemia 451 *

Hyperaldosteronism 456*

Hyperaminoacidemia 96

Hyperammonemia 209*

Hyperbilirubinemia 214*

Hyperbilirubinemia, neonatal 215*
Hypercalcuria, idiopathic 202*

Hypercholesterolemia 215*

Hypercholesterolemia, familial 218*

Hyperglycemia 120

Hyperglycinemia 238

Hyperkalcemia 442 *

Hyperkalemia 345

Hyperkalemic periodic paralysis 286

Hyperlipidemia 208*

Hyperlysinemia 89

Hypersensitivity $436^{*}$, 437*

Hypersensitivity, delayed $437 *$

Hypertriglyceridemia 219*

Hypertrophy 244

Hyperuricemia 204*

Hypodipsia $458^{*}$

Hypoglycemia 280, 295, 377*

Hyponatremia 345

Hypophosphatasia 450*

Hypopituitarism 25, 36, 207*

Hypopituitary 459*

Hypoproteinemia 318

Hypothyroidism 459*

Hypoxemia $379 *$, 444*, 467*, 479*

Hypoxemia, chronic $444 *$

Hypoxia 46, 201*, 456*, 468* $469 *, 473 *$

Hypoxic splenic circulation $465 *$

Hyvarinen, M.A. 470*

$\operatorname{IgA} 71$

IGO, R.P. 474*

Immaturity $446 *$, 479*

Immune response 500

Immunity $371 *, 372 *, 373 *$, $376^{*}, 461^{*}$

Immunity, cellular $437 *$

Immunity, rubeola $460 *$

Immunization $376^{*}$

Immunization, nasal 436*

Immunoglobulin $460 *, 462 *$

Immunoglobulin, IgA 435*, $437 *$

Immunoglobulin, IgE 435*, $436 *$

Immunoglobulin, IgG 437*

Immunoglobulin, IgM 437*

Immunoglobulins 437*

Immunology 194*, 207*, 437*

Impetigo $448 *$

Inanition 379*, 449*

Indian, American, Papago 474*

Infancy $214^{*}$

Infant $204^{*}, 207 *, 377^{*}$

Infant mortality 194

Infant mortality rate 373 *

Infants 175, 202*, 208*, 216*, $218 *, 262,373 *, 378 *$ $379 *, 380^{*}, 440 *, 466^{*}, 470 *$ $472 *, 474 *, 476 *, 477 *$, $479 *, 481 *$

Infants, low birth weight $375^{*}$

Infants, neglected $474^{*}$

Infants, premature $389,473 *$ 
Infection $461 *, 462 *, 464 *, 500$ Infection, Escherichia coli 463* Infection, fetal $460 *, 461 *$ Infection, intrauterine $375^{*}$ Infection, $M$.pneumoniae $460 *$ Infection, viral $462 *$

Infectious hepatitis $463 *$

Insensible water loss $201 *$, 473* Insulin 120, 206*, 280, 295, $453 *, 456 *, 471 *, 472 *$

Intestinal absorption 412 Intestinal function $477 *$ Intestine 218*

Intragenic complementation $376^{*}$

Intrahepatic cholestasis $215^{*}$

Intrauterine blood transfusions $378 *$

Intravascular coagulation $372 *$

Iron deficiency 244

Iron metabolism 218*

IsHIZAKA, K. 435*

ISRAELS, L. G. 465*

JAcobson, C. B. $372 *, 377 *$

JANKUS, E.F. 377*

JARMAKANI, J.M. 444*

Jaundice $476^{*}, 481^{*}$

JEAN, R. 207*

JEGK, D. L. $450 *$

JENNER, M.R. 380*

Jensen, J. 448*

Johnson, C.A. 464*

JoHNSON, L.H. 481*

Johnson, O. C. 103

JoRDAN, G. M. 464*

Jose, P.A. 446 *

KABACK, M. M. 440*, 465*

KAISER, G. A. 444*

KAJII, T. 441*

KAPLAN, E.L. 460*

KaPLAN, S. $464 *$

Kaplan, S. L. 25, 36,380*, 480*

KARLSSON, B. $211^{*}$

KATTWINKEL, J. 436*

KATZ, M. 482*

KaUfMaN, D. B. 448*

KAYE, R. 295

KAZEMI, H. 379*

КEKOMÄKI, M. 209*

Kennedy, C. 481 *

KenNeLl, J.H. 473*

KenNy, F.M. 457*, 458*

Ketoacidosis $451 *$

$\alpha$-Ketoglutarate 446*

11-Ketopregnanetriol 145

Ketosis 206*, 238

Ketotic hyperglycinemia 439*

KHEDER, J. 467*

Kidney 7, 257, 280, 372* $376^{*}, 378 *, 435^{*}, 445 *$, $448 *, 468 *$

Kidney, cystic disease $448 *$

Kidney, function $446^{*}, 450^{*}$, $455^{*}$

Kidney, metabolism 446*, $447 *, 448^{*}$

Kidney, transplantation 449*
KILDEBerg, P.A. 447*

KIM, O.K. 455*

KIM, Y.J. 450*

KIRKLAND, J. 475*

KISKER, C.T. 468*

Kitterman, J.A. 469*

KLAUS, M.H. $473^{*}$

KleIN, R. 286, 444*, 456*

Kleinfelter's syndrome 25

Kleinman, L. I. 468*

KlingBerG, W. G. 452*

KLyman, G. 470*

KNELSON, J.H. 201*

KNIKER, W.T. 448*

KNITTLE, J.L. 373*

KOCHEN, J.A. 466*

Kolobow, T. 470*

KoprowsKi, H. 482*

KorNHAUSER, D. 120

KoROTZER, J. 436*

Korsch, B. M. 449*

KOSKINEN, K. R. 472*

Kotas, R. V. 201*

Kouvalainen, K. 208*

KowalewsKI, S. 215*

KowARSKI, A. 129 455*

Krauss, A.N. 479*

KRETCHMER, N. 55

Krivit, W. 377*

KROVETZ, L.J. 445*

Krugman, S, 372*

KRZESKA, I. 500

KugEL, R.B. 187

Kunnast, M 202*

Kulovich, M. V. 352

KulvinsKas, C. 448*

KumaR, L. 435*

Kunzman, E.E. 295

Kuplic, L.S. 372*

Kwashiorkor 135, 213*

Lactate 46, 202*

Lactic acid 451*

LAhey, M.E. 457*

LALEZARI, P. 437*

LAMPERT, F. 204*

LANDING, B. 437*

LANDOWNE, R.A. 352

LANG, D. J. $462 *$

LANGE, K. 376*

LANZKOWSKY, P. 468*

LAPEY, A. 457*, 478*

LASSMAN, H. B. 82

Lean body mass 268

LEBOVE, F. 202*

Lecithin 352

LEFFLER, A.T. 451*

LEIDY, G. 463*

Leigh's encephalopathy 204*

LeIKIN, S. 436*

LEONARD, A.S. $377 *$

LEONARD, C.O. $440 *$

LEONARD, L. $482 *$

LEQUIRE, V.S. 469*

LERMAN, S.J. $460^{*}$

Lesch-Nyhan disease 204*

Lesch-Nyhan syndrome 452*

Leukemia $461^{*}$

Leukemia, acute $467 *$
Leukemia, chronic myelogenous $467 *$

Leukocyte 203*, 206*, 461*, $462 *$

Leukocytes 405, 437*, 438*

Leukocytosis, cyclic $467 *$

LEUNG, L-S. 461*

LEvin, E. Y. 465*

LEVINE, A.S. $461 *$

LEVISON, H. 477*, 478*

LEVITSKY, L. L. $472 *$

LEVY, R.I. 439*

LEWY, J.E. 446*

LEwY, P.R. 448*

Liddle's syndrome $455^{*}$

LIEBERMAN, E. 449*

Liebhaber, H. 373 *

LINDBERG, T. 211 *

LINDBLAD, B. $216 *$

LiNDENMANN, J. 213*

LindQuist, B. $210^{*}$

LINDSTEDT, S. 216*

LINSAO, L. 318

Lipid metabolism 219*

Lipid synthesis $453 *$

Lipids 352

Lipocytes $373^{*}$

Lipolysis $473^{*}$

$\beta$-Lipoprotein $440 *$

$\alpha, \beta$-Lipoproteinemia 219*

$\beta$-Lipoproteinemia, hypo $376 *$

Lipoproteins, high density $439 *$

Lipoproteins, serum 218*

Liv, C. 463*

Liver 157, 203*, 204*, 214* $215 *, 217^{*}, 328,337,377^{*}$, $438 *, 442 *, 452 *, 463 *$

Liver disease $216 *, 476 *$

Liver, lysosomal diseases 57 , 216*

LLOYD, J.K. 218*, 219*

LLOYD-STILL, J.D. 464*

LOEB, H. 209*, 214*

LOGAN, A. G. 446*

LOLEKHA, S. 437*

LORAs, B. 145

LOUGH, M.D. 165

LOVEJOY, F.H.,Jr. 464*

LOVRIEN, E. W. 440*

Lowy, C. 280

Lubin, B. H. 466*, $476 *$

Lugo DE RIVERA, C. $477 *$

LUNDE, M.N. 461*

Lung $201^{*}, 352,378^{*}, 469 *$, $470^{*}, 478^{*}$

Lung, capillary flow 472*

Lung, chronic obstructive disease $477 *, 479 *$

Lung, function $379 *, 477 *, 479 *$

Lupus erythematosus $435 *$

Lustik, B. 448*

Luteinizing hormone 25, 36, 175, 456*

Lux, S.E. 439*

Lymphedema 377*

Lymph, flow, hepatic $377 *$

Lymphocyte $71,204 *, 437 *$

Lymphocytic choriomeningitis virus 194 
LXNGH, M.J. 477*

Lyon, G. M.,Jr. 454*

Lysergic acid diethylamide 377 *

Lysine 89

Lysine-ketoglutarate reductase 89

Lysosomal membrane 493

MAatela, J. 208*

Macrocephaly 481*

MADDAIAH, V.T. 457*

Magnesium 210*

Magnesium, metabolism 452*

MAHONEY, M.J. 374*

MaKowsKr, E. L 441* 471*

Malabsorption $476^{*}, 477^{*}$

Malabsorption, glucosegalactose $211^{*}$

Malabsorption, intestinal 212*

Malabsorption, protein $210^{*}$

Malabsorption, sugar 210*

Malate dehydrogenase 328

MALM, J.R. 444*

Malnutrition 213*, 318, 345, 375*

Maltase 476*

Mandible, hypoplasia $441^{*}$

Manganese 157

MANN, L. I. 46

MARGOLIS, J.A. 475*

MARKOWITZ, M. 371*

MARSHALL, R.E. 440*

Masera, G. 203*

MASTRANGelo, R. 467*

MATALON, R. 372*

Maternal blood 76

Maternal hemorrhage 82

Mathieu, H. 202*

MATHIEU, M. 204*, 214*

MAtsaniotis, N. 203*

Matthews, L. W. 165

Maturation 462*

Maturation, skeletal 458*

MAURER, H. M. 465*

MAY, G.D. 437 *

McIntosh, R. M. 448*

McNamara, H. 476*

Measles 460*

Measles, antibodies 482*

Measles, antibody $482^{*}$

Measles, virus $482 *$

Medearis, D.N., Jr. 461*

MEDOVx, H. 208*

Medroxyprogesterone acetate 458*

Meeuwisse, G. 211*

Melatonin 456

MeLby, J. 456*

MeLish, M.E. 378*

Mellits, E.D. 157

Meliman, W.J. 371*

MELTZER, D. $453^{*}$

Meningitis, bacterial 4.74*

Menkes, J.H. 480*

Menstruation 36

Mental retardation 238, 318, $452 *, 454 *, 474 *$

Mentzer, W. G. $465 *$

Meschia, G. 445*, $471^{*}$
Mestyan, J. 202*

Metabolic acidosis $377 *$

Metabolic homeostasis 472*

Metabolism 14

Metabolism, amino acid $454 *$

Metabolism, carbohydrate $477 *$

Metcoff, J. 446*, 448*

Methionine 238, 337, 454*, 476*

$\beta$-Methylcystathionine 205*

Methylmalonicaciduria $374 *$

Methylmalonyl-CoA mutase 374*

Middleton, F., Jr. 435*

Mrgeon, C.J. 129, 455*

Milgram, E.A. 443*

MrLic, A. M. B. 257

Miller, L. 462*

Miller, W.W. 444*

MILNER, R.D. G. 213*

Mirowskr, M. 445*

Mist, therapy $478 *$

Mitochondria 244, 328

Moe, P. J. 218*

Moes, G.A.F. 318

Mohyuddin, F. 447*, 450* $451 *$

Monkus, E.F. 453*

Monosodium glutamate 204*, 373*

Montagnier, L. 462*

MONTGOMERY, J.R. 463*

Montgomery, R 461*

Moreau, P. 206*, 214*

MorpHIs, L. G. 446 *

Morphogenesis $440^{*}$

Morrison, A.D. 466*

Morrison, L. 479*

MORRISON, T. 76

Morrow, G., III 451*, 453*, 480*

Morse, C.W. 474*

Mortality, perinatal 473 *

Moser, H.W. 451*

Moshang, T.,Jr. 175, 455*

MosIER, H.D. Jr, 459*

Mothering 473*, 474*

Mother-offspring infection 194

Motoyama, E. K. 478*

Mucopolysaccharides $372 *$

Mucopolysaccharidosis 206*

Mucosa, small intestine 212*

MukherJee, A. 375*

Mull, M. 229

Munschauer, R. W. 459*

Muranyi, L. 202*

Murillo, G. J. 71

Murphy, M. L. 461*

Muscle 268, 286, 444*

Muscle cell mass 135

Myelin 203*, 480*

Myerburg, R.J. 444*

Myocardial hypertrophy $444 *$

Myocarditis $377 *$

Myotonia 286

NACHMAN, R.L. 468*

NADLER, H.L. 376* 439*

NAEYE, R.L. 257, 375*, 473*
Nathan, D. G. 465*, 476*

National Nutrition Survey 103

Nelson, N. M. 479*

Nelson, T.E. $441^{*}$

Neonatal $211 *$

Nephritis 378*, 460*

Nephritogenic antigen $376^{*}$

Nervous system, adrenergic $444^{*}$

Nesbit, M.E. 467*

Neter, E. 500

Neuroblastoma 454*

Neurolipids 96

Neuromuscular disease 480*

Neutropenia $437 *$

Neutrophil $467 *, 493$

Newborn 7, 14, 82, 120, 202* $203 *, 218 *, 379^{*}, 437^{*}$, $442 *, 448 *, 453 *, 466 *$ $469 *, 471 *, 472 *, 479 *$, $480 *, 481 *$

Newborn, feeding 473*

Newborn, low birth weight $470^{*}, 472^{*}, 473^{*}, 478^{*}$

Newborn, rabbits $203 *$

Newborns 165, 201*, 202* $203 *, 217 *, 229,257,280$, $295,376^{*}, 412,422$

Newcomb, R.W. 435*, 436*

Nrcholson, J.F. 389,398

Nicolopoulos, D. 203*

NIELSON, J. 205*

NIKKARI, T. 208*

NisWANDER, J.D. 474*

NISWANDER, K.R. 82

Nitroblue tetrazolium dye $462 *, 463 *$

Nitrogen metabolism 389, 398

Nogrady, B. 378*

Nonketotic glycinemia $480 *$

Nordio, S. 206*

Noren, G.R. 377*

Norepinephrine 453*

Norman, A. 214*

Novak, M. $453^{*}$

NOYEs, W.D. 468*

Nucleic acid synthesis $481 *$

Nutrition $473^{*}, 474 *$

OBERKIRCHER, O.R. 500

Obesity 268, 373*

O'Brien, D. 96

Ockerman, P.A. 216*

OGRA, P.L. 371*

OH, W. 378*, 470*

O'Hara, P. $441 *$

Olafsson, A. 202*, 215*

Oliver, W.J. 463*

OLSON, W.H. 379*

O'Neal, R.M. 103

OPPENHEIM, J.J. 436*

Optic nerve dysplasia 480*

ORSINI, A. 217*

OrT, M. $446^{*}$

Oskr, F.A. 371*, 444*, 466*, $470^{*}$

Osteochondritis $452 *$

Osteopetrosis 218*

Osteoporosis 202*

OUTERBRIDGE, E.W. 378* 
528

Overall, J.E. 63

Oxidative metabolism $379 *$

Oxygen 202*, 471*

Oxygen, therapy 469*, 470*

OzoA, N. 459*

Page, L.A. $450 *$

Pancreas, pseudocyst 208*

Pantlitsahko, M. 205*

Paramyotonia 286

Parathyroid extract $210 *$

Parent-child interaction $475 *$

PARK, B.H. 463*

PARKe, J.C., Jr. 376*

PARKeR, M.L. 459*

PARMLEy, T. H. 440*

PARra, A. 268

Partin, J. G. $376 *$

Parvinen, T. 208*

Pate, J.E. 474*

PAthak, A. 479*

PAunier, L. 210*

PAyne, F.E. 482*

Pearson, H.A. $372 *$

Peckham, G.J. 444*

Penicillin 436

Perheentupa, J. 209*

Perin, D. M. 449*

Periodic breathing 478*

Peritoneal dialysis 202*, 449*

Perletti, L. 203*

Perlstein, P.H. 472*

Permeability, capillary 446*

Perrimond, H. 217*

Peter, G. 464*

Petit, P. 214*

Pfeiffer, R. 207*

Pham-Hư-Trung, M. T. 207*

Pharmacogenetics 187

Phenobarbital 214*

Phenobarbitone 215*

Phenylalanine 96, 318

Phenylketonuria 318

Phribis, R. H. 469*

Phocomelia $441 *$

Phosphate 2,3-diphosphoglycerate $470^{*}$

6-Phosphogluconic dehydrogenase 405

Phospholipids 217*

PIEL, C.F. 378*

Piepsz, A. 214*

Pierce, J.E. 470*

Pierre-Robin syndrome 441*

Pierson, M. 202*

PikAar, N.A. 206*

Pincus, T. 435*

Pinsky, L. 438*

Pipecolic acid 89

Pituitary $175,456 *, 457 *$

Pituitary gland 257

Pituitary, growth retardation 459*

Placenta 46, 82, 129, 345, 379*, $380^{*}, 442 *, 455^{*}, 471^{*}$

Placenta, artificial $470 *$

Placental transfer 76

Plasma binding 187

Plasma clearance 412
Index Volume 4

Platelets 379*, 464*, 465*

Pneumococcus 461*

Poisoning 475*

Poliovirus $371 *$

Polonovski, C. 212*

Polyol metabolic pathway 466*

Polysaccharides 451*

Popich, G.A. 441*

Porphyria, congenital erythropoietic $465^{*}$

Porte, D., Jr. 456*

Posterior fossa cysts $481^{*}$

Postnatal malnutrition 14

Potassium 286, 345

Potter, D.E. $449 *$

Poverty $375 *$

Powell, E. 438*

Prasad, A. L.N. 295

Precocious puberty 309

Pregnancy 377*, 445*

Pregnanetriol 145

Pregnanolone 471*, 480*

Premature 82, 201*, 202*, 203*, 442*

Premature infants $14,76,89$, 389, 398, 412

Prematures 202*

Prematurity $375 *$

Progesterone 76

17-OH Progesterone 380*

Progressive septic granulomatosis 493

L-Proline 447*

Propionyl-CoA carboxylase 439*

Propper, R.D. 446*

Prosencephalon 480*

Protein-calorie malnutrition 135

Protein intake 398

Protein synthesis 157, 389

Proteins, fibrinolytic $470 *$

PRUdENT, L. M. 479*

Pryles, C. V. $448 *$

Pseudoaldosteronism 455*

Pteroylglutamic acid 412, 422

Puberty 25, 459*

Pulmonary physiology 165

Pulmonary resistance 7

Pupene, M. B. 89

Purine synthesis 204*

Pyelonephritis 448*, 450*

Pyridoxine 205*, 441*, 454*

Pyrimidine biosynthesis 55

Pyrudine nucleotide ratios 466 *

Pyruvate 46, 451*

Pyruvate kinase 209*

Pyruvate kinase deficiency 465*

RABE, E.F. $481 *$

Race 375*

RAGHMELER, M. 376*

RADEL, E. 437*

Radioactive iodinated human serum albumin (RISA) $481^{*}$

Radioiodine 374*

RaIHA, N. 203*, 442*, 443*

RANKIN, J.H.G. 445*
RASHKIND, W. J. 444*

RAssin, D. 337, 453*

RAYE, J.R. 469*

ReBA, R. G. 268

REDMAN, W. 463*

REEs, E.P. $479 *$

Renal clearance $446 *$

Renal disease 378*

Renal gluconeogenesis $446 *$

Renal hemodynamics 7

Renin 457*

Renin release $457 *$

REPLOGLe, R. L. 445*

Reouien 206*

Respiration 63, 469*, 478*

Respiration, fetal $471 *$

Respiratory distress syndrome $82,202 *, 378 *, 469 *, 470 *$

Reticulocytes $465 *$

Reticuloendotheliosis 437*

REY, J. 213*, 219*

REY, J.P. 210*

REYNoLDs, J.W. 455*, 458*

Rezvani, I. 457*

R-factor $472 *$

Rh erythroblastosis $378 *$

Rheumatic fever $207 *, 371 *$, 460*

Rheumatoid arthritis 436*

RiGHARD, C.A. 468*

Richmond, J.B. 374*

Rickets 318

Rickets, vitamin D resistant $451 *$

Ridler, M. A. C. 441*

RIEU, D. 207*

Rigatto, H. 472*, 478*

RikKeRs, H. 442*

Riseborough, E. P. 379 *

RNA 157, $442 *$

RoBbins, J.B. $376 *$

Robinson, S.H. 465*

RoBinson, W.A. 467*

RockeL, T.H. 481*

RODRIGUES, L.P. $376^{*}$

Rodriguez DE CURET, H. 477*

Rogers, S. 452*

Romeo, G. 465*

Romero, T. $443^{*}$

Root, A.W. 175, 456*

ROSENBERG, D.M. 373*

Rosenberg, L.E. 374*, 439*, 454*

ROSENFIELD, R.L. 459*

Ross, A. 462*

Rottauwe, H.W. 215*

Round heart disease $377^{*}$

ROWE, R.D. 445*

Rowland, L.P. 480*

Rubella $373^{*}$

Rubella vaccine $373 *$

Rubella virus vaccine $372 *$

Rubeola 460*, 482*

Rubeola, virus $482 *$

Rubin, M.I. 500

Rubino, A 477*

RudOLPH, A. M. 443*

Ruitton, 206*

Ruiz, G. 446* 
Index Volume 4

Russ, R.D. 456*

RÜTER, E. 217*

SAGEL, I. 376*

SAHLER, O.J.Z. 474*

Salicylate 187

Salicyluric acid 187

Salte, B. 204*

SAlvador, H.S. 471*

SANDBERG, D.H. 76, 262

San Filippo syndrome 451 *

Sarcosine 450*

Satterthwaite, H.S. 379*

Scalp vein needles $464 *$

SahaEFer, A.E. 103

SCHAFER, I.A. 438*

SCHAFFNER, F. 205*

SGHÄRER, H. 217*

SCHARER, K. 446*

SCHEDL, H.P. 477*

Saheinman, J.I. 372*

Schieken, R.M. 444*

SchIFF, D. 280

SCHIMPFF, R. M. 207*

SChlegel, R.J. 405, 462*

Schmerler, A. $462 *$

Schmickel, R. 439*

SCHNEERSON, R. 376*

SCHNEIDER, A.J. 476*

SCHNEIDER, J.M. 471*

SchneIdman, K. 453*

Schroeder, W.A. 466*

SChröter, W. 217 *

SaHubert, W.K. 376*, 452*

SahulHofF, C. $373 *$

SCHULMAN, I. $379 *, 464 *$

Schulman, J.D. 379*, 452*

Sahultz, R.B. 268

Schwartz, A. D. $372 *$

Sahwartz, A.L. 203*

Schwartz, E. $476^{*}$

SchwARTZ, R. 120

Scoliosis 379*

Scort, C.R. 440*

SGRIVER, G.R. 447*, 450*, $451 *$

Scully, K.J. 439*

SEASHore, M.R. 454*

Secretory piece 71

Sedation 165

SEEGMILleR, J.E. 379*, 452*

SEELY, J.R. 451*

SEgAL, S. 447*, 452*

SELL, S.H.W. $474^{*}$

Semenza, G. 213*

Septicemia $464^{*}$

SERENI, F. 203*

Sereni, L. 203*

Severi, F. 207*

Sexual development 175

Sexual maturation 25, 36

ShankLin, D.R. 469*

ShanNon, D. C. 379 *

ShaRP, H.L. 377*

Sheep 46

ShePARD, T.H. $441 *$

SherMETA, D. W. 129, 201*

SHINER, M. 214*

SHMERLING, D.H. 214*
SHOHET, S. B. $466 *, 476 *$

Shojania, A. M. 412,422

SHORE, N. A. 437*

SHWAGHMAN, H. 456*, 477*

Sickle cell anemia $372 *, 475 *$

SIEGEL, S.E. 461*

SigneR, E. 215*

SigSTAD, H. 377*

StLKaLNS, G.1. 450*

Simkó, X. 208*

Srmmons, J. M. 449 *

Simopoulos, A. P. 457*

Singer, D.B. $437 *$

SINGER, H. S. $438 *$

Stzonenko, P. G. 25, 36

ŞöLrn, S. 217*

Skeletal development $373 *$

Skin, temperature $472 *$

Skjaeveland, A. 218*

Slotkoff, L. M. 446*

Smith, A.L. 379*

Sмгтн, C.H. 328

Smith, D.W. $440 *, 441 *$

SMith, G. F. 44I*

SMrTH, J.D. 45I*

SmirH, N.J. 474*

Sodium 202*, 345, 457*

Sodium, erythrocyte 456 *

Sodium flux $445^{*}$

Sodium metabolism 145, 465*

SOELDNER, S.J. 456*

Sokoloff, L. 379*, 481*

SOLER, J.A. 468*

SOLOMON, G.E. 337

SOODALTER, J. 479*

South, M.A. 463*

SOYKA, L.F. 471*, 480*

SPENGER, R.P. $372 *$

SpIEgel, A. M. 458*

SPITZER, A. $450 *$

Spleen $372 *, 461 *$

SPRINGER, D. 345

Sprunt, K. 463*

Stahlman, M.T. 469*

STALEY, N.A. $377 *$

STambaUgh, M. G. 374*

Staphylococcal scalded skin syndrome $378^{*}$

Staphylococcus 378*, 463*

STARK, A. 373*

Steatorrhea $476 *$

SteInHART, J.M. 500

STERKY, G. 219*

STERN, L. 378*

Steroid 458

Stevenson, J.E. 377

St.Geme, J.W.,.Jr. 460*

STIEHM, E.R. 372*, 470*

Stinson, D.A. $479 *$

STOWERS, C. $473^{*}$

Strandvix, B. 214*

Streptococcus $376^{*}, 448 *, 460 *$

Sturman, J.A. 205*, 337, $442 *, 453 *$

Subacute sclerosing panencephalitis (SSPE) 482*

Sucrase-isomaltase, intestinal 213*

Sulfonylurea $458 *$
Sumida, G. $453^{*}$

Surface activity 352

Surfactant 201*

SutherLand, J. M. 472*

Svoboda, D. J. 463*

SWANSTRÖM, S. 469

Systemic lupus erythematosus 378*

SzAL, G. 461*

TALAmo, R.C. $477 *$

TALLAN, H. 453*

TAlner, N.S. $443 *$

Tangier disease 219*, 439*

TAPPER, A. J. 455*

TARLOW, M.J. 211*

TAYLOR, P.M. 446*, 479*

Tay-Sachs disease 206*

Temperature, skin $472 *$

TERAMO, K. $443 *$

TER Meulen, V. 482*

Testicle 25

Testosterone 309

Thermal effects, cooling $472 *$

Thermogenesis $473 *$

Thiamine $451^{*}$

Thoracic gas volume 165

Thrombocytes 229

Thrombocytopenia 464*

Thyroid $457 *$

Thyroid cancer $374 *$

Thyrotoxicosis $374 *$

Thyrotropin $457 *$

Thyroxin $480 *$

Thyroxine $\left(T_{4}\right) 457 *$

TICKNOR, W. $478^{*}$

Timmermans, J. 206*

Tommasi, M. 204*

Tonsils $371 *$

TOOLEY, W.H. 469*

Torres-Pinedo, R. 477*

TOWELl, M.E. 471*

Toxin 378*

Toxoplasmosis 461 *

Transformation, lymphocyte 436*

Transformation, nuclear $441 *$

Transport, amino acid $477^{*}$

Transport, potassium $445^{*}$

Transport, sodium $445 *$, $448^{*}, 478^{*}$

Transsulfuration $337,442 *$

Travis, L. B. 450*

Travis, S.F. 466*

Troelstra, J.A. 238

Trygstad, C.W. 372*

Trypsinogen deficiency, pseudo $212 *$

Tsoulos, N.G. 471 *

TURNER, M.D. 378*

Twins $467 *$

TY, A. 376*

Tyrosinemia $216 *, 337$

Tyrosine transaminase 203*

UeHLing, D. T. 372*

UGLIENGO, 206*

UlLELAND, C. 474*

Umbilical circulation 46

Umbilical cord $380^{*}, 471^{*}$ 
UNDERWOOD, B.A. 475*

Urea 389

Urea metabolism 398

Uridine kinase 55

Urinary tract 500

Urine 280

Urine data 103

Uroporphyrinogen III

cosynthetase $465^{*}$

USHER, P. 445*

USHER, R.H. 469*

VAcCARo, R. 207*

Vaccine, $H$. influenza type b $376^{*}$

Vaccine, influenza $436^{*}$

Vaccine, Pseudomonas 461*

VAINSEL, M. 214*

VALENCA, L. M. 379*

VAN DE KAMER, J.H. 210*

VAN DE Velde, G. 209*

Vanillylmandelic acid (VMA) 203*

Van Pelt, L.F. 460*

Varicella 462*

Vector analysis $445 *$

VEGTER, D. 439*

Ventilation 165

VERNIER, R. L. 448*

VERT, P. 202*

VEST, M. 215*

Virus 435*, 462*

Virus disease $378 *$

Virus, mumps $460 *$

VISAKORPI, J.K. K. 209*

Visser, H.K.A. 309

Vitamin A $475 *$

Vitamin $\mathrm{B}_{6} 441$ *
Vitamin $B_{12} 374 *$

Vitamin $\mathrm{D}_{3} 442 *$

Vitamin E $475^{*}$

Vitamin E dificiency $466^{*}$

von THADDEN, H. 462*

Voth, D.W. 463*

Vovan, L. 217*

VOYER, L.E. $448 *$

VuREK, G.G. 470*

WADA, Y. $453 *$

WALD, M. 473*

WANNAMAKER, L. W. 448*, $460^{*}$

WARD, A. B. 448*

WARKANY, J. 431

WARSHAW, J. B. 244, 442*

Water 14

Water, intercellular 286

WATSON, D. W. 479*

WEBB, W.W. 474*

WEIJERS, H.A. 210*

WEIL, W.B.,Jr. 365

Weintraub, D. H. 82

WEIPPL, G. 205*

WEISSER, K. 202*, 215*

WEKSLER, M. B. 468*

WeLdon, V.V. 455*

WENNBERG, R.P. 474*

WEST, E.F. 450*

Whang-Peng, J. 436*

WHELAN, D.T. $450^{*}$

WhITE, J. G. 467*

White villus disease $376 *$

WhitTen, C.F. 475*

WILMINK, R. 309

WILSON, J. G. 427
Wilson's disease 217*

WINEGRAD, A.I. 377*, 466*

Winters, R.W. 447*

Wrtmer, G. 202*

WOLFE, L.S. 438*

WolfF, O.H. 218*

WOLFSON, S.L. $372 *$

WOLPERT, S. M. 481*

Wong, P.W.K. 454*

WONG, S.R. 448*

Wong, V.G. 379*

Wood, D.E. 478*

WOODY, N. G. 89

WRANNE, L. 217*

WYLER, F. 202*

Wyss, M. 210*

Xanthou, M. 203*

YANG, M. C. $462 *$

YoshidA, T. 446*, 448*

Young, L. S. 461*

YounGBLOOD, L. 461*

Younoszar, M.K. 477*

YuAN, S. 7

ZAPOL, W.M. $470 *$

ZELSON, J.H. 372*

ZetTERSTRÖM, R. 214*, 216*

ZigAs, G.J. 478*

Zinc 157

ZIPURSKY, A. $465^{*}$

ZirbeL, C.L. 379*

ZIRING, P.R. 372*

Zoster immune globulin 462*

ZUelzer, W.W. 467*

ZWEYMÜLLER, E. 201* 